\title{
Resectable Dedifferentiated Liposarcoma
}

National Cancer Institute

\section{Source}

National Cancer Institute. Resectable Dedifferentiated Liposarcoma. NCI Thesaurus.

Code C150607.

Dedifferentiated liposarcoma that is amenable to surgical resection. 